\title{
Sex Differences in Keloidogenesis: An Analysis of 1659 Keloid Patients in Japan
}

\author{
Chikage Noishiki · Yoshiaki Hayasaka · Rei Ogawa
}

Received: June 28, 2019 / Published online: October 4, 2019

(C) The Author(s) 2019

\begin{abstract}
Introduction: Keloids are a cutaneous fibroproliferative disorder. Despite the fact that keloids are relatively common lesions, the statistics of patient with keloids especially sex difference remain unknown. To better understand it, we conducted an extensive cross-sectional analysis of a large cohort of patients with keloids $(n=1659)$. The study showed for the first time that female sex may be an inherent keloid risk factor.
\end{abstract}

Methods: This cross-sectional study of 1659 consecutive patients with keloids who attended a plastic surgery outpatient clinic in Japan in 2014 analyzed age at keloid onset, age at the first medical examination for keloid, and the influence of sex on these variables.

Results: In both male and female patients, the keloids were most likely to start in puberty and there was no significant difference in the mode value for age of onset (16 vs. 20 years). Though

Enhanced Digital Features To view enhanced digital features for this article go to https://doi.org/10.6084/ m9.figshare.9778679.

C. Noishiki · R. Ogawa ( $₫)$

Department of Plastic, Reconstructive and Aesthetic

Surgery, Nippon Medical School, Tokyo, Japan

e-mail: r.ogawa@nms.ac.jp

Y. Hayasaka

Center for Medical Education, Nippon Medical

School, Tokyo, Japan female patients were twice as prevalent as male patients at nearly all onset ages, female patients predominated over male patients with a gender ratio of 2.7:1 in cases of onset before the age of 15 years. Moreover male and female patients did not differ in terms of the mean \pm SD duration between keloid onset and the first medical examination. This finding shows that female patients do not get their keloids examined earlier than male patients. These observations together suggest that female sex may promote early keloid development due to physiological, not social, reasons.

Conclusion: This is the first report to suggest that female sex may drive keloidogenesis because of physiological reasons. Individuals were most likely to seek a medical examination almost 10 years after onset, regardless of sex. These findings provide new insight into the importance of sex in the development and progression of keloids. Future studies should address the influence of sex hormones on the keloid.

Keywords: Cross-sectional study; Female; Keloid; Wound healing

\section{INTRODUCTION}

Keloid is a cutaneous fibroproliferative disorder that is the result of an impaired wound healing course that leads to persistent inflammation in 
the wound and continuous deposition of collagen fibers. Keloids are widely seen as being highly intractable to therapy, although we have had considerable success recently with a treatment regimen composed of surgery, radiation, and postoperative steroid tape application [1].

Many lines of research suggest that keloid is driven by multiple factors, including hereditary predisposition. These factors may also include systemic factors such as hormones: it is well known that keloids worsen with pregnancy $[2,3]$ and hypertension [4-6]. Conditions that increase the blood levels of inflammatory cytokines such as interleukin-6 (IL-6) may also promote the development of severe keloids [7].

Previous studies have reported on standard treatment of keloids as a combination of several modalities, namely, surgery, followed by radiation, and prolonged daily use of corticosteroid injection/tape/plaster/ointment $[8,9]$, cryotherapy, compression therapy, stabilization therapy, 5-fluorouracil (5-FU) therapy, Nd:YAG Laser [10], and carbon dioxide laser [11].

Despite being a relatively common skin lesion, many aspects of keloid remain unclear, including its prevalence and how many of the patients with keloids experience keloid-related morbidity, undergo a medical examination for it, and undergo treatment. Indeed, only a few papers have reported statistical analyses of patients with keloids [12-14]. In particular, the age at keloid onset is poorly understood. To address these gaps in knowledge, we conducted an extensive cross-sectional analysis of a large cohort of patients with keloids $(n=1659)$ who presented to our clinic for a medical consultation about their keloid(s) in 2014. The aim of this study was to investigate the age at keloid onset, the age when the patient underwent their first medical examination for keloid, and how sex influenced these variables.

\section{METHODS}

\section{Selection of Study Subjects}

The study cohort consisted of all consecutive patients with one or more keloids who underwent consultation for their keloid(s) at the
Nippon Medical School Hospital in the 2014 business year (1 April 2014-31 March 2015). All patients were diagnosed with keloid on the basis of their history and the clinical findings.

\section{Study Variables}

The following data were collected from all patients: sex; ethnicity; age, race, body mass index, smoking habit, and medical history (hypertension, hyperlipidemia, diabetes mellitus, allergy, and family history of keloid) at the index visit to our clinic; age at keloid onset; age at the time of the first medical examination for keloid.

\section{Statistical Analyses}

Continuous variables were expressed as mode and/or mean \pm standard deviation. Categorical variables were expressed as numbers (\%). The cohort was divided into two groups on the basis of sex and the male and female patients were compared in terms of the variables described above by independent Student's $t$ test. The male and female patients were compared in terms of the duration between keloid onset and the first medical examination by cross tabulation. All statistical analyses were performed using Microsoft Excel 97-2003 (Microsoft, USA) and SPSS statistical software (IBM, USA). Two-tailed $p$ values of less than 0.05 were considered to indicate statistical significance.

\section{Compliance with Ethics Guidelines}

This cross-sectional study was conducted at the Department of Plastic, Reconstructive, and Aesthetic Surgery of Nippon Medical School Hospital, Tokyo, Japan. The study protocol was approved by the institutional review board of Nippon Medical School Hospital (approval no. 26-11-406). The study was conducted in accordance with the tenets of the Declaration of Helsinki. 


\section{RESULTS}

\section{Demographic and Medical History Characteristics of Total Cohort at Index Visit to Clinic}

Between April 2014 and March 2015, 22,339 consecutive patients were examined at the Department of Plastic, Reconstructive, and Aesthetic Surgery at Nippon Medical School Hospital. The 22,339 patients were $0-101$ years old; 9480 (42.4\%) were male and 12,859 $(57.6 \%)$ were female. Thus, female patients predominated over male patients with a gender ratio of $1.4: 1$.

Of these 22,339 patients, 1659 patients underwent the examination because they had one or more keloids. This group constitutes one of the biggest keloid cohorts in Japan to date. All patients were Asian. The ages of the patients with keloids at the index visit ranged from 0 to 83 years. Female patients predominated over male patients with a gender ratio of $2: 1$. This was also true for nearly all decennary age groups. However, there were two exceptions, namely, the 0-9-year-olds, where the gender ratio was $1.7: 1$, and the $10-19$-year-olds, where the gender ratio was 1.2:1 (data not shown). Table 1 summarizes the demographic and clinical characteristics of all male and female patients in this cohort. The two groups did not differ significantly in terms of age at the index visit, race, BMI, hyperlipemia, diabetes, allergy, or family history. However, compared to the female group, the male group had significantly more hypertension $(12.3 \%$ vs. $5.6 \%, p=0.005)$ and significantly more smokers $(15.0 \%$ vs. $5.4 \%, p<0.001$ ) (Table 1).

\section{Age at Keloid Onset}

Of the 1659 patients in the cohort, 369 were excluded because they did not know their age at keloid onset. The remaining 1290 patients were 0-78 years old and 403 and 887 were male and female, respectively. Thus, the female patients were twice as prevalent as male patients $(68.8 \%$ vs. $31.2 \%)$. The male and female patients did not differ significantly in terms of mean \pm SD age of onset $(24.5 \pm 14.4$ vs. $25.1 \pm 15.1$ years $)$ or the mode value for age of onset (16 vs. 20 years) (Fig. 1a). Figure $1 \mathrm{~b}$ shows the age at keloid onset of the male and female patients in this sub-cohort. In both male and female patients, keloids were most likely to start in puberty. Female patients were twice as prevalent as male patients at nearly all onset ages, including before the age of 15 years, where they were 2.7 -fold more prevalent.

\section{Duration Between Age of Onset and Age at First Medical Examination for Keloid}

Of the 1659 patients in the overall cohort, 864 were excluded because they did not know their age at the first medical examination for keloid and/or when their keloids first arose. Of the remaining 795 patients, 267 and 528 were male (Fig. 1d) and female (Fig. 1e), respectively $(33.6 \%$ vs. $66.4 \%)$. The male and female patients did not differ significantly in terms of mean \pm SD age at index visit $(34.1 \pm 16.1$ vs. $35.3 \pm 15.0$ years) or the mode value for age at index visit (22 vs. 21 years). Figure 1c shows that the male and female patients did not differ in terms of the mean \pm SD duration between keloid onset and the first medical examination $(9.5 \pm 10.3$ vs. $10.1 \pm 10.0)$.

Figure $1 \mathrm{~d}$ and e are scatter diagrams showing the relationship between the age at onset ( $\mathrm{x}$ axis, where age is expressed in 5-year units) and the duration between onset and the first examination (y-axis, where duration is expressed as $0,1,2,3$, and 4 years followed by 5 -year units) for the male and female patients. The color of the boxes indicates the frequency of the patients at each point of the grid. The male and female patients exhibited very similar scattering. In particular, the most common scenario for both male and female patients (indicated by the warm colors) was that their keloid(s) started at age 15-24 and they had their keloid(s) examined 5-14 years later. This finding shows that female patients do not get their keloids examined earlier than male patients. 
Table 1 Characteristics of the 1659 patients with keloids who underwent consultation for their keloid(s) in the Nippon Medical School Hospital in 2014

\begin{tabular}{llll}
\hline Patient characteristic & Male $(\boldsymbol{n}=\mathbf{5 6 0})$ & Female $(\boldsymbol{n}=\mathbf{1 0 9 9})$ & $\boldsymbol{p}$ value \\
\hline Age, years & & & \\
Mean (SD) & $35.0(16.0)$ & $36.0(14.9)$ & 0.238 \\
Mode & 22.0 & 22.0 & - \\
Sex, $n$ (\%) & $560(33.8)$ & $1099(66.2)$ & - \\
Asian ethnicity, $n$ (\%) & $560(100)$ & $1099(100)$ & \\
Body mass index ${ }^{\mathrm{a}}$ & & & 0.602 \\
Mean (SD) & $22.8(3.3)$ & $21.3(3.3)$ & $0.005^{*}$ \\
Mode & 23.5 & 20.8 & 0.480 \\
Hypertension, $n(\%)$ & $69(12.3)$ & $61(5.6)$ & 0.253 \\
Hyperlipidemia, $n$ (\%) & $22(3.9)$ & $31(2.8)$ & $<0.001^{*}$ \\
Diabetes, $n(\%)$ & $10(1.8)$ & $8(0.7)$ & 0.077 \\
Current smoking, $n(\%)$ & $84(15.0)$ & $59(5.4)$ & 0.36 \\
Allergy, $n$ (\%) & $213(38.0)$ & $504(45.9)$ & $232(21.1)$ \\
Family history, $n(\%)$ & $100(17.9)$ & & \\
\hline
\end{tabular}

$S D$ standard deviation

${ }^{*}$ Significant at $p<0.05$

${ }^{a}$ BMI is calculated as weight in kilograms divided by height in meters squared

\section{DISCUSSION}

This is the first report that extensively analyses relationships among the age at keloid onset, the age at first medical examination, and sex.

\section{Female Sex May Be a Keloid Risk Factor}

Several previous studies of cohorts of patients with keloids show that the male to female ratio is almost $1: 2[12,15,16]$. However, it is widely believed that male and female patients are equally likely to develop keloids and that patients with keloids who come to the hospital for treatment are more likely to be female simply because of social factors, namely, that female individuals care more about their appearance, are less resistant to medical examinations $[17,18]$, and are more likely to get their ears pierced than male individuals [19]. Several reports have described ambulatory care visits to hospital outpatient departments in the USA [20-23]. The female visit rate was higher than the rate for male patients overall. However, for patients under 15 years of age, the visit rate in boys was higher than that of girls.

However, our study casts doubt on this notion and indeed suggests that at least in childhood-onset patients, the female gender is an inherent systemic factor that strongly promotes keloid onset or progression. First, the patients in our large hospital cohort $(n=1659)$ were indeed twice as likely to be female overall (Table 1) and Fig. 1b shows that this female predominance is present at nearly every onset age (Fig. 1b). Of particular interest is that female patients tended to predominate strongly in the childhood onset group (i.e., onset before the age of 15 years): girls were 2.7 -fold more prevalent than boys in this group. However, our analysis 

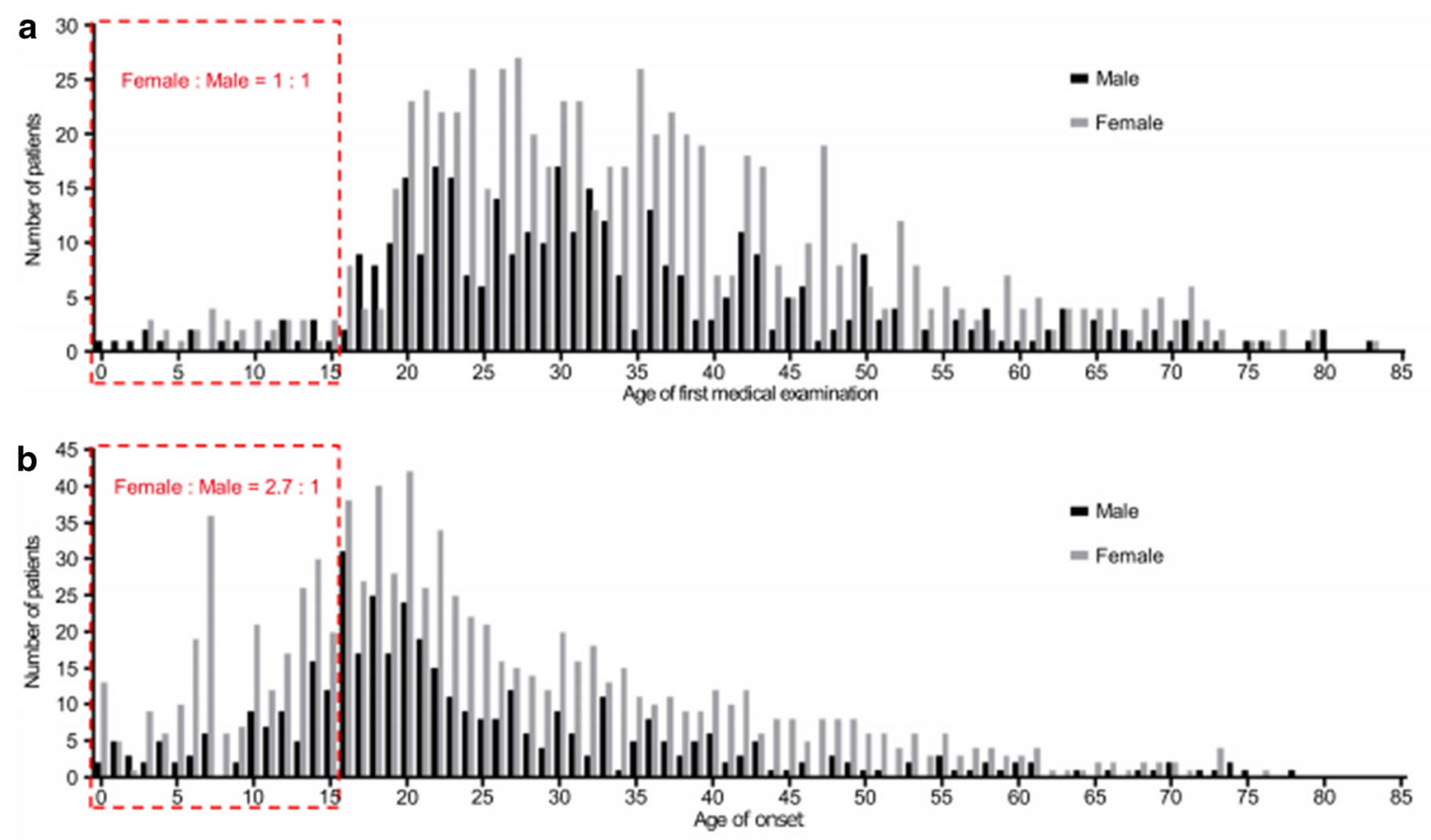

C

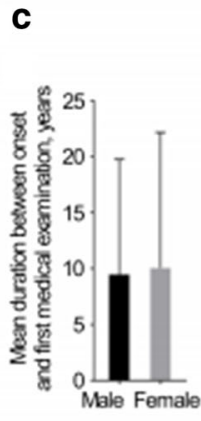

d
Age of onset (Male)

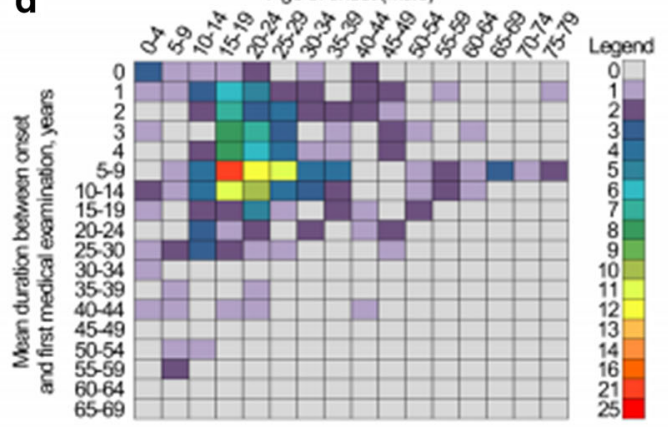

e Age of onset (Female)

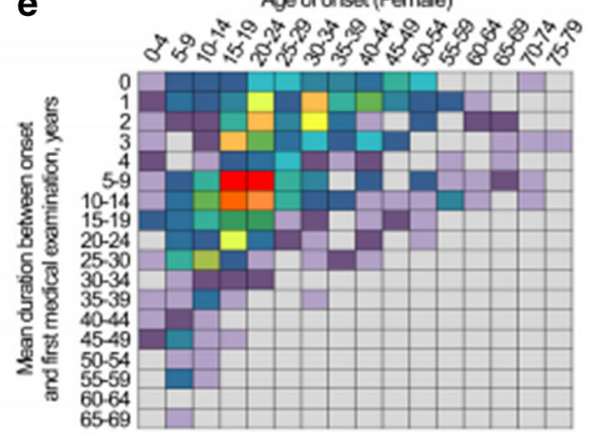

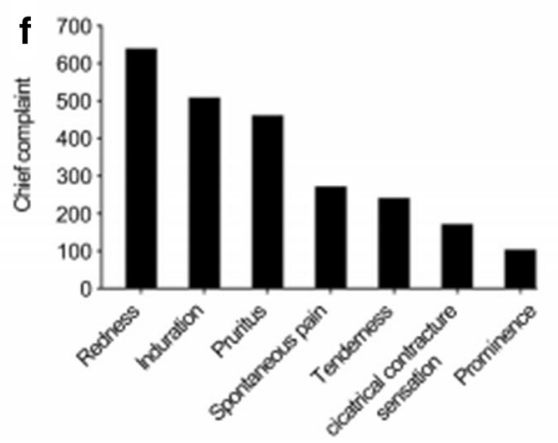

Fig. 1 Age of the patients at a their first medical examination for keloid $(n=1072)$ and b keloid onset $(n=1290)$. c Mean duration between keloid onset and the first medical examination. Cross-tabulation analysis showed there were no statistically significant differences between the male and female patients in terms of this variable. The bar whiskers show standard deviation.
Relationship between the age of keloid onset ( $\mathrm{x}$-axis) and the duration between onset and the first medical examination (y-axis) in male patients (d) and female patients (e). f Histogram showing the most common chief complaints $(n=2405)$ of the 1659 patients with keloids. In $\mathbf{a}-\mathbf{c}$, male and female patients are depicted by black and gray bars, respectively 
of the age at the first medical examination of the patient's keloids showed that in the childhood-onset patients who visited a medical practitioner before the age of 15 years, boys were equally as prevalent as girls. Thereafter, female patients abruptly became much more prevalent (Fig. 1a). This pattern probably reflects the fact that before the age of 15 years, the family of the patient was initiating the visit to the doctor, without showing preference for gender. Therefore, our data suggest that at least up to the age of 15 years, female gender may be an inherent keloid risk factor.

Interestingly, our analyses also cast doubt on one of the social factors that are believed to drive the female predominance in patients with keloids seen in hospitals, namely, that female individuals care more about their appearance and are therefore more likely to seek keloid treatment than male individuals. However, Fig. 1c shows that the two sexes did not differ in terms of mean duration between onset and the first medical examination. Moreover, the scatter diagrams in Fig. 1d, e show that for both sexes, onset was most common in puberty and young adulthood (15-24 years) (Fig. 1b) and that the patients in this group were most likely to seek a medical examination 5-9 years after onset, regardless of sex.

Another piece of evidence that supports the notion that female sex promotes keloidogenesis, at least in childhood, relates to vaccinationinduced keloids. Thus, even though boys and girls are equally likely to be vaccinated on the upper arm in childhood and vaccinations are a very common trigger of upper arm keloids, the female patients in our cohort were significantly more likely than male patients to have upper arm keloids (data not shown).

The mechanism driving female predominance in patients with juvenile-onset keloids is unknown but may reflect sex hormones. This is supported by the fact that keloids worse in pregnancy $[3,24]$. A sex-related genetic predisposition may also contribute to female predominance. Further studies are needed to determine the mechanisms underlying female predominance in juvenile-onset keloids in vitro.

\section{Sex Does Not Influence Keloid Onset Age or Age at First Examination}

We did not observe any differences between male and female patients in terms of keloid onset age: the mode values for keloid onset age in the male and female patients were 16 and 18 years, respectively. Notably, a previous report describing the age of onset in 418 patients with keloids reported an older onset age, namely, 19-29 years [14]. In addition, we observed that in both sexes, onset age exhibited a single peak, namely, in puberty (Fig. 1b). We also did not observe any differences between male and female patients in terms of age at the first medical examination: in both sexes, the age at the first medical examination for keloid exhibited a single, albeit broad, peak in the 20-30s (Fig. 1a). Notably, a previous study of 135 patients with keloids reported that the age at the first medical examination exhibited two peaks, one at 20-39 years and the other at 60-79 years [15]. These differences between our study and others may reflect the smaller sample sizes in the other studies and/or regional differences between hospitals in terms of the type of patients in their catchment area.

\section{Study Limitations}

This study was descriptive, which means that it may be subject to selection and information bias that may affect the reliability of the results, namely, race, etiology, and consciousness about appearance.

\section{CONCLUSIONS}

This study showed that female individuals may be more prone to keloids than male individuals because of physiological reasons, especially with regards to juvenile-onset keloids. Furthermore, in subsequent reports, we will further analyze and discuss keloids in patients with juvenileonset disease and hypertrophic scars. Future pathology, histological, and gene-expression studies on how sex and other factors, including hypertension [4-6] and endothelial dysfunction 
[25], affect keloid development and progression are warranted.

\section{ACKNOWLEDGEMENTS}

Funding. No funding or sponsorship was received for the publication of this article. All authors had full access to all of the data in this study and take complete responsibility for the integrity of the data and accuracy of the data analysis.

Authorship. All named authors meet the International Committee of Medical Journal Editors (ICMJE) criteria for authorship for this article, take as a whole, and have given their approval for this version to be published.

Author Contributions. Chikage Noishiki conceived and designed the research; Chikage Noishiki carried out the experiments; Chikage Noishiki and Yoshiaki Hayasaka analyzed the data; Rei Ogawa supported the study; Chikage Noishiki wrote the manuscript.

Disclosures. Chikage Noishiki, Yoshiaki Hayasaka, and Rei Ogawa have nothing to disclose.

Compliance with Ethics Guidelines. This cross-sectional study was conducted at the Department of Plastic, Reconstructive, and Aesthetic Surgery of Nippon Medical School Hospital, Tokyo, Japan. The study protocol was approved by the institutional review board of Nippon Medical School Hospital (approval no. 26-11-406). The study was conducted in accordance with the tenets of the Declaration of Helsinki.

Data Availability. This datasets generated during and/or analyzed during the current study are available from the corresponding author on reasonable request.

Open Access. This article is distributed under the terms of the Creative Commons Attribution-NonCommercial 4.0 International
License (http://creativecommons.org/licenses/ by-nc/4.0/), which permits any noncommercial use, distribution, and reproduction in any medium, provided you give appropriate credit to the original author(s) and the source, provide a link to the Creative Commons license, and indicate if changes were made.

\section{REFERENCES}

1. Ogawa R, Akaishi S, Kuribayashi S, Miyashita T. Keloids and hypertrophic scars can now be cured completely: recent progress in our understanding of the pathogenesis of keloids and hypertrophic scars and the most promising current therapeutic strategy. J Nippon Med Sch. 2016;83(2):46-53.

2. Moustafa MF, Abdel-Fattah MA, Abdel-Fattah DC. Presumptive evidence of the effect of pregnancy estrogens on keloid growth: case report. Plast Reconstr Surg. 1975;56(4):450-3.

3. Park $\mathrm{TH}$, Chang $\mathrm{CH}$. Keloid recurrence in pregnancy. Aesthetic Plast Surg. 2012;36:1271-2.

4. Arima J, Huang C, Rosner B, Akaishi S, Ogawa R. Hypertension: a systemic key to understanding local keloid severity. Wound Repair Regen. 2015;23:213-21.

5. Ogawa R, Arima J, Ono S, Hyakusoku H. Case report total management of a severe case of systemic keloids associated with high blood pressure (hypertension): clinical symptoms of keloids may be aggravated by hypertension. Eplasty. 2013;13:e25.

6. Ogawa R. High blood pressure (hypertension) may influence the results of clinical trials for scar and keloid treatments. Plast Reconstr Surg. 2013;132:1074e-5e.

7. Quong WL, Kozai Y, Ogawa R. A case of keloids complicated by Castleman's disease: interleukin-6 as a keloid risk factor. Plast Reconstr Surg Glob Open. 2017;5:e1336.

8. Ogawa R. Keloids and hypertrophic scars can now be treated completely by multimodal theraphy, including surgery, followed by radiation and corticosteroid tape/plaster. Scars. 2019. https://doi.org/ 10.5772/intechopen.84178.

9. Goutos I, Ogawa R. Steroid tape: a promising adjunct to scar management. Scars Burn Heal. 2017;3:2059513117690937. 
10. Koike S, Akaishi S, Nagashima Y, Dohi T, Hyakusoku H, Ogawa R. Nd:YAG laser treatment for keloids and hypertrophic scars: an analysis of 102 cases. Plast Reconstr Surg Glob Open. 2014;2(12):e272.

11. Tenna S, Aveta A, Filoni A, Persichetti P. A new carbon dioxide laser combined with cyanoacrylate glue to treat earlobe keloids. Plast Reconstr Surg. 2012;129(5):843e-4e.

12. Omori Y, Akaishi S, Ogawa R, Hyakusoku H. Analysis of regions where keloids to occur. Off J Jpn Scar Workshop. 2010;4:112-5.

13. Ogawa R, Okai K, Tokumura F, et al. The relationship between skin stretching/contraction and pathologic scarring: the important role of mechanical forces in keloid generation. Wound Repair Regen. 2012;20(2):149-57.

14. Tosa M, Murakami M, Hyakusoku H. Study on age at onset of keloids. Scar Manag. 2012;6:69-70.

15. Tanaka Y, Kusumoto K, Takemoto T, Hihara M, Tanaka Y. Assessment of 135 cases of keloids experienced to date at the Kansai Medical University hospital. Scar Manag. 2012;6:66-8.

16. Sun LM, Wang KH, Lee YC. Keloid incidence in Asian people and its comorbidity with other fibrosis-related diseases: a nationwide population-based study. Arch Dermatol Res. 2014;306(9):803-8.

17. Bertakis KD, Azari R, Helmas LJ, Callahan EJ, Robbins JA. Gender differences in the utilization of health care services. J Fam Pract. 2000;49(2):147-52.
18. Wang Y, Hunt K, Nazareth I, Freemante N, Petersen I. Do men consult less than women? An analysis of routinely collected UK general practice data. BMJ Open. 2013;3:1-7.

19. Ly N, McCaig LF. National hospital ambulatory medical care survey: 2000 outpatient department summary. Adv Data. 2002;327:1-27.

20. Hing E, Middleton K. National hospital ambulatory medical care survey: 2001 outpatient department summary. Adv Data. 2003;338:1-26.

21. Hing E, Middleton K. National hospital ambulatory medical care survey: 2002 outpatient department summary. Adv Data. 2004;345:1-36.

22. Middleton K, Hing E. National hospital ambulatory medical care survey: 2003 outpatient department summary. Adv Data. 2005;366:1-36.

23. Quaranta A, Napoli C, Fasano F, Montagna C, Caggiano G, Montagna MT. Body piercing and tattoos: a survey on young adults' knowledge of the risks and practices in body art. BMC Public Health. 2011;11:774.

24. Mustoe TA, Cooter RD, Gold $\mathrm{MH}$, et al. International clinical recommendations on scar management. Plast Reconstr Surg. 2002;110:560-71.

25. Noishiki C, Takagi G, Kubota T, Ogawa R. Endothelial dysfunction may promote keloid growth. Wound Repair Regen. 2017;25:976-83. 